$\xi=-1$

\title{
Pre filtering techniques for face recognition based on edge detection algorithm
}

\author{
Sheela Rani *, Vuyyuru Tejaswi, Bonthu Rohitha, Bhimavarapu Akhil \\ Department of Computer Science and Engineering, Koneru Lakshmaiah Education Foundation \\ *Corresponding author E-mail: akhilbhimavarapu@gmail.com
}

\begin{abstract}
Recognition of face has been turned out to be the most important and interesting area in research. A face recognition framework is a PC application that is apt for recognizing or confirming the presence of human face from a computerized picture, from the video frames etc. One of the approaches to do this is by matching the chosen facial features with the pictures in the database. It is normally utilized as a part of security frameworks and can be implemented in different biometrics, for example, unique finger impression or eye iris acknowledgment frameworks. A picture is a mix of edges. The curved line potions where the brightness of the image change intensely are known as edges. We utilize a similar idea in the field of face-detection, the force of facial colours are utilized as a consistent value. Face recognition includes examination of a picture with a database of stored faces keeping in mind the end goal to recognize the individual in the given input picture. The entire procedure covers in three phases face detection, feature extraction and recognition and different strategies are required according to the specified requirements.
\end{abstract}

Keywords: Face Recognition; Digital Image; Face Detection; Feature Extraction.

\section{Introduction}

Recognition of face has been the fascinating topic in various areas of research. The area of biometrics [1] uses the concept of face recognition to identify the persons involved in crime etc. In face recognition these steps are followed

A) Capturing the image.

B) Face-detection.

C) Feature-extraction

D) Face-recognition.

a) The digital data of the image -capturing a person's digital data using a camera,

b) Perform face detection -(detecting the human faces from the given digital image). The places in the image where there is a significant or a sharp change in the brightness is termed as an edge. In the given image face is recognised or detected by using the principals of Edge-detection.[6]. Generally the face detection is performed by extracting the intensity of each pixel and grouping the elements, then identifying the similar pixels and detecting the face.

c) Feature extraction (this helps in identifying the similar images by considering various images of the same person). In feature extraction we generally detect the edges in the given image. Various edge detection techniques that are primarily used are, Sobel, Prewitt, Canny etc., Comparative analysis of edge detection methods has showed that canny is the most powerful edge detector [2].

d) Finally face recognition (comparing the acquired features with the images in the database and identifying them). There are a lot of uses of facial recognition technique. It is widely used in security areas, social networking like Facebook, and in mostly personalized marketing. Recognition of face is used by Facebook to give suggestions to user in tagging his/her friends in the image, this works by storing the various characteristics of the person in the database and thereby comparing the features. Now-a-days many apps regarding face recognition like face-lock screen, face vault, Oasis face, Nametag were also developed.

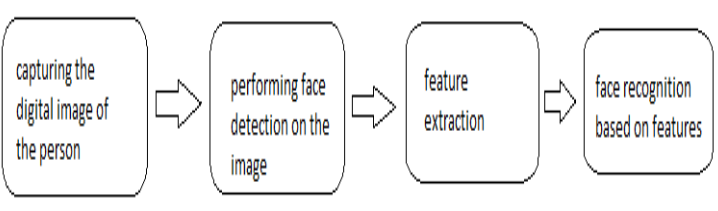

Fig. 1: Process Flow.

Image processing uses simple techniques that can be utilized for the printed copies like printouts and photos. Picture experts utilize a scope of essentials of understanding while at the same time utilizing these visual methods. Image processing isn't quite recently confined to range that must be examined however on information of expert. Association is another imperative mechanism for image processing through visual strategies. So investigators apply a blend of individual learning and guarantee information to processing of image. Image processing is emphatically identified with PC vision and PC designs.

\section{Literature survey}

The Author A.D.Chitra et. al [15] has discussed about canny operator. The author said that the canny operator is the best of all other gradient based approaches and LoG filters. Canny operator detects all the edges clearly and localizes the edges perfectly. To get more accurate result using canny operator adaptive calculation of the threshold can be made based on gradient magnitude and its direc- 
tion with high threshold the canny operator gives better performance and more continuity.

[2] In this paper the author has performed some experimental analysis on the images to know the behavior of 0-crossing operators and gradient operator on the capability of detection of edges. After applying various techniques Canny 0perator was found to identify edges those were both strong and weak. Canny approach is more appropriate for detecting edges when compared to Laplacian of Gaussian (LoG).

[8] In this paper the author discusses about all the edge detection algorithms both Gradient and Laplacian of Gaussian operators. Gradient based operators are very sensitive to any noise present. Laplacian based operators are better than Gradient based operators. Canny edge detection method gives good results and performs well when compared to remaining edge detection operators.

[10] In this paper the author has discussed about various edge detection methods to extract information from the images in a less time duration based on some properties such as computation time and capability of reducing noise. According to the author canny approach takes more time for giving the results.

[18] In this paper the author has taken a dataset of different images and performed the face recognition using the software mat-lab. The whole process was divided into 6 steps. In the first step the edge-map of the given input image using the operator sobel. In the second step the $\mathrm{S}, \mathrm{H}$ values are computed, then some mathematical computation is performed and the pixel was determined to be a skinned or non-skinned pixel, various regions of the image are identified and different parameters concerning that image are calculated. The problem of illumination has been overcome in this paper [14] in this paper some hardware implementation for the recognition of face was done. Firstly the edge detection was performed on the given set of images using the operator canny, then Gaussian mask was used on the images. Coming to hardware im- plementation a pic microcontroller with some display of lcd was used to identify the human faces. The result was highly affected by variations in lighting and some differences in the angle of the pose given in the image.

\section{Methodology}

Face recognition frameworks have been directed now for right around 50 years. Facial recognition is one of the explores in zone design recognition and PC vision because of its various down to earth applications in the range of biometrics, Information security, get to control, law implementation, keen cards and reconnaissance framework. The principal huge scale utilization of recognition of face was done in Florida. Face recognition is such a testing yet fascinating issue that it has pulled in scientists who have distinctive foundations: brain research, pattern recognition, neural systems, PC vision, and PC illustrations. There are many methods available for face recognition the available methods are divided into 3 methods

\section{- Holistic-Methods}

- Feature-based Methods

- Hybrid-Methods

Facial recognition was dealt with as a $2 \mathrm{D}$ design recognition issue. The separations between critical points where used to perceive known appearances, e.g. measuring the separation between the eyes or other vital focuses or measuring diverse points of facial segments. In any case, it is important that the face recognition frameworks to be completely programmed accompanying strategies are utilized.

\begin{tabular}{|c|c|c|c|}
\hline METHODS & HOLISTIC METHODS & FEATURE BASED METHODS & HYBRID METHODS \\
\hline OVERVIEW & $\begin{array}{l}\text { In holistic approach, the complete face region is taken into } \\
\text { account as input data into face catching system. One of the } \\
\text { best example of holistic methods are Eigenfaces }\end{array}$ & $\begin{array}{l}\text { In this methods local features such as eyes, nose and } \\
\text { mouth are first of all extracted and their locations and } \\
\text { local statistics (geometric and/or appearance) are fed } \\
\text { into a structural classifier }\end{array}$ & $\begin{array}{l}\text { Hybrid face recognition systems use a } \\
\text { combination of both holistic and feature } \\
\text { extraction methods. Generally } 3 D \text { Images } \\
\text { are used in hybrid methods. The image of a } \\
\text { person's face is caught in } 3 D \text {, allowing the } \\
\text { system to note the curves of the eye } \\
\text { sockets, for example, or the shapes of the } \\
\text { chin or forehead. }\end{array}$ \\
\hline DESCRIPTION & $\begin{array}{l}\text { (1) The first stage is to insert a set of images into a database, } \\
\text { these images are names as the training set } \\
\text { (2) The second stage is to create the eigenfaces. } \\
\text { (3) When the eigenfaces have been created, each image will be } \\
\text { represented as a vector of weights. } \\
\text { (4) The system is now ready to accept entering queries. } \\
\text { (5) The weight of the incoming unknown image is found and } \\
\text { then compared to the weights of those already in the system. }\end{array}$ & $\begin{array}{l}\text { A big challenge for feature extraction methods is } \\
\text { feature "restoration", this is when the system tries to } \\
\text { retrieve features that are invisible due to large } \\
\text { variations, e.g. head Pose when we are matching' a } \\
\text { frontal image with a profile image. Distinguishes } \\
\text { between three different extraction methods: } \\
\text { I. Generic methods based on edges, lines, and curves II. } \\
\text { Feature-template-based methods } \\
\text { III. Structural matching methods that take into } \\
\text { consideration geometrical Constraints on the features. }\end{array}$ & $\begin{array}{l}\text { The image of a person's face is caught in } \\
3 D \text {, allowing the system to note the curves } \\
\text { of the eye sockets, for example, or the } \\
\text { shapes of the chin or forehead. Even a face } \\
\text { in profile would serve because the system } \\
\text { uses depth, and an axis of measurement, } \\
\text { which gives it enough information to } \\
\text { construct a full face. The 3D system usually } \\
\text { proceeds thus: Detection, Position, } \\
\text { Measurement, Representation and } \\
\text { Matching. }\end{array}$ \\
\hline
\end{tabular}

Fig. 2: Review of Face Recognition Methods.

\section{Face detection}

Face Detection is a technique to identify human faces in a visual scene. In this step we process in the following way. i) The grey level image is considered and all the available human eye parts are identified by detecting the valley regions present in image.

ii) The head movement which causes the shirring effect and the unequal illumination or the brightness which causes the lightning effect is corrected for every person in image.

iii) The projection of Eigen faces is used for measuring the fitness value of every person. The persons with high fitness 
value are identified by repeating the same process. The face symmetry of verified.

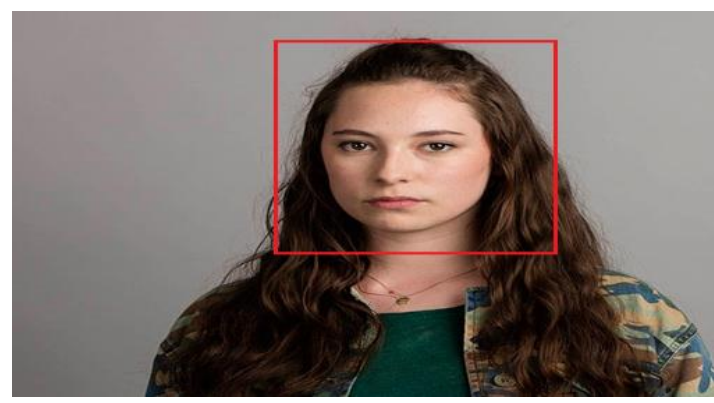

Fig. 3: An Image Containing a Human Face.

In fig. 3 we see the image of a girl. We the input as the plain image and the face is detected/recognised from the given image. Even if many persons are present in the picture their faces in the input image can be detected to generate the output image.

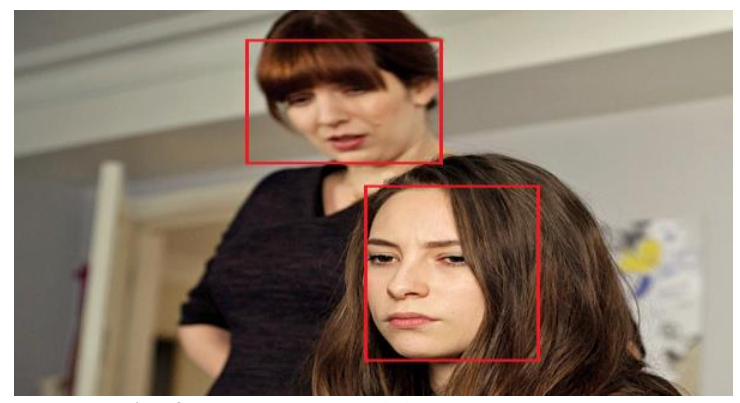

Fig. 4: An Image Containing A Human Faces.

In fig.4. There are two persons in the given image and their faces were detected as the output. Some of the applications in which the face detection is used as a primary concern are Facial motion capture, Facial recognition, photography, marketing in a very innovative like a camera is installed in our personal television sets facial detection the software application then measures the race, the gender, and age range of the face. As the information regarding the person is gathered, the related advertisements are played regarding their race/gender/age.

\section{Feature extraction}

feature extraction begins from an underlying arrangement of measured information and fabricates inferred values (features) proposed to be useful and non-excess, encouraging the ensuing learning and speculation steps, and at times prompting better human elucidations. Feature extraction is identified with dimensionality reduction. When the information to a calculation is too extensive to possibly be handled and it is suspected to be excess (e.g. a similar estimation in feet and meters, or the repetition of pictures exhibited as pixels), at that point it can be changed into a decreased arrangement of features (additionally named a feature vector). Deciding a subset of the underlying features is known feature selection. The chosen features are relied upon to contain the applicable data from the information, with the goal that the coveted undertaking can be performed by utilizing this decreased representation rather than the total initial information.

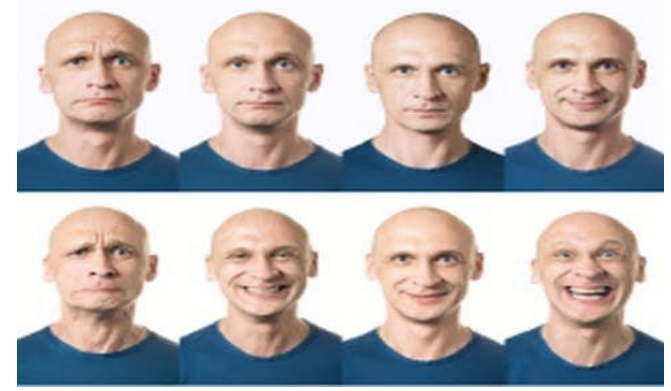

Fig. 5: Feature Extraction.

In fig 5 Different poses of the person are considered to depict that all the images have the features. Feature Extraction retrieves same features even for different images of the same person. Feature extraction internally uses the edge detection techniques to retrieve the features.

A) Edge detection

Edge detection is typically analysed as the way of distinguishing the focuses on the picture where the brightness changes pointedly or formally. The focuses at which picture shine changes forcefully are commonly sorted out into an arrangement of curved gathering portions usually termed as edges. Edge detection is the central apparatus for picture division. It divides a computerized picture into numerous regions or pixels [6]. The 3 fundamental steps are:

a) Filtration: Each picture is related with a couple of significant values for their intensities, random enhancements that were done on the values/qualities can cause disturbances usually known as noise. Noise may lead to troubles in viable edge discovery; subsequently picture ought to be separated with a specific end goal to lessen the disturbances in the content which can cause the damage to edge strength. Additionally, this is also termed as Smoothening.

b) Enhancement: moving forward those nature for picture may be coined as Enhancement. It ought to transform a picture which may be that's only the tip of the iceberg preferable Furthermore more suitableness over first. A channel will be connected so as on enhancing the nature for edge of a picture.

c) Detection: Many techniques were employed to figure out which points would be the edge focuses, which edge pixels ought to acknowledged as disturbance and uproot those disturbances. This transform determines the frameworks associated with an object and the foundation of the picture. Identification from claiming edge serves in picture reconstruction, information layering also division of picture. Variables are convoluted for choice about a edge identification driver incorporate edge coordination, commotion surroundings [3].
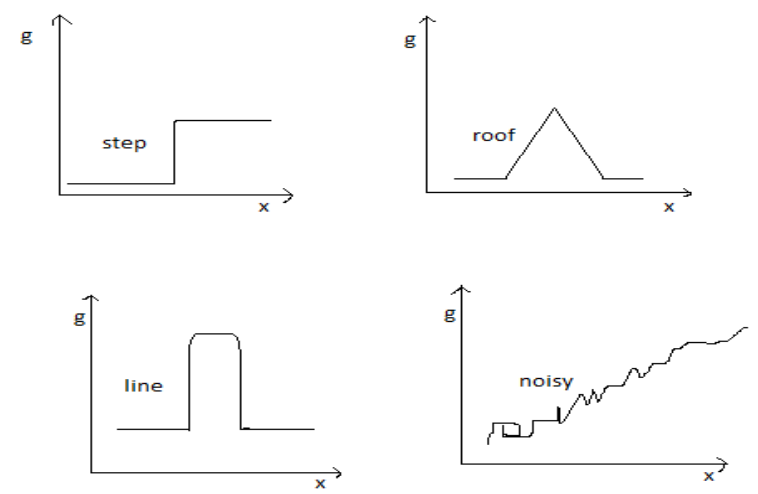

Fig. 6: Graphs Indicating Noise Environment.

Edge Detection is utilized for the most part to remove the information about the picture e.g. picture upgrade and area of question exhibit in the picture, and picture sharpening and furthermore their shape, estimate. Edge identification is an important task of picture segmentation. The adequacy of many picture handling likewise 
PC vision errands relies upon the flawlessness of identifying significant edges. The way toward characterizing and setting sharp discontinuities in a picture is called edge-detection. The procedure of arranging and putting sharp discontinuities in a picture is known as the edge identification. The edge portrayal of a picture altogether diminishes the amount of information to be forms, yet it holds basic data in regards to the states of articles in the scene [5]. Edge identification is a fundamental instrument to examination scenes. An edge indicator is a high-pass channel that can be utilized to separate the edge focuses in a picture. An edge is a nearby change in the force of the picture. It is related with an intermittence of power of the picture. The primary reason for edge discovery in a computerized picture is to check the force changes. Edge discovery is generally utilized for distinguishing changes in dark level pictures, recognizing segregated focuses and thin lines in light of the fact that separated focuses and thin lines does not often happen in most down to earth pictures for any picture handling application, question acknowledgment and protest location edge recognition is a vital pre-preparing step [6].Corrupted images are also called as noisy images. They are difficult to analyse and detect. In many fields of computerized picture preparing edgedetection is utilized. Most regularly the edge discovery is utilized as a part of picture division, enlistment, identifying proofs, and so forth. Picture dividing is the way toward isolating a computerized picture into various areas. A right division of a picture is acquired by utilizing thresholding, area developing, or edge identification theorems. The steps to perform edge detection are: Filtering, Detection, Edge localization.

a) Filtering: To improve the performance of edge detector this step involves filtering the image for noise reduction.

b) Detection: All the edge points that are possible candidates to become edge point are extracts in the step.

c) Edge localization: It comprising an edge. It selects the points of the edge from the candidate edge points that are true members of a set of points.

Edge-detection is a procedure that recognizes the nearness and area of edges constitute by sharp changes in shading power or shine of a picture. It likewise alludes to the way toward distinguishing and finding sharp discontinuities in a picture. The discontinuities are fast changes in pixel force which recognize limits of articles in a scene. The edge discovery process serves to improve the investigation of pictures by fundamentally lessening the measure of information to be prepared, while in the meantime protect helpful basic data about question limits. Edge detection, portions the question while sifting the commotion while safeguarding the auxiliary properties of the picture. Edge location winds up plainly troublesome if there should be an occurrence of uproarious pictures [9].

B) Edge-detectors

An edge can be depicted as a critical change in nearby intensity/brightness, for the most part connected with a move in either the picture brightness or the principal subsidiary of the picture intensity level.

Angle based edge location calculations utilize primary subsidiary operation. The highlights of inclination based edge identification frameworks are they are basic and can identify

Edges along a few introductions. In spite of the fact that the weaknesses are their affectability to disturbance [13].Well known edge identifying calculations are Sobel, Prewitt, Robert, and Krisch.

The procedure for edge recognition is arranged into two classifications; first is angle based and second is laplacian's based. In the inclination based technique edges are identified by taking the principal arrange subsidiary of the picture. The angle size can be utilized to appraise a measure of edge quality. Inclination greatness has the properties of neighbourhood online maxima helps in figured nearby edge introduction which with the slope bearing. In the Laplacian based strategy, picture can be utilized to process the second request subsidiary articulation which has a zero intersection. For the most part, edges are ordinarily found by looking zero intersection of a non-direct differential articulation. Ordinarily for edge location a pre-handling step Gaussian smoothing is connected which is usually a refining stage [4].

The most usual edge detection techniques that are used for differentiating are:

I). Sobel edge-detection

The sobel's operator plays out an estimation which is two dimensional spatial slope estimation to highlight on the spatial recurrence relating to the edges. It is fundamentally used to locate the total inclination greatness in input dim scale picture at each point. This is by and large like the Robert's operator. [8]

\begin{tabular}{|c|l|l|}
\hline-1 & 0 & +1 \\
\hline-2 & 0 & +2 \\
\hline-1 & 0 & +1 \\
\hline
\end{tabular}

GX

\begin{tabular}{|l|l|l|}
\hline+1 & +2 & +1 \\
\hline 0 & 0 & 0 \\
\hline-1 & -2 & -1 \\
\hline
\end{tabular}

Gy
Fig. 7: Sobel Mask.

Sobel's edge-detection will help on giving some smoothing impact on the picture therefore it also takes attention of the noise within the image. This strategy extracts all edges in an image, irrespective of its direction. A Sobel edge detection technique provides both a differencing and smoothing effect. This incorporates a couple of $(3 \times 3)$ convolution parts. One specific piece is basic and the other turned by $90^{\circ}$. The pieces can be utilized on the information picture, to produce singular estimations of the angle segment toward every path.

The sobel's edge discovery strategy is like those of the Robert's cross edge identification administrator. The plan of sobel's and Robert edge identification administrator are same, however the key huge distinction is that the got picture is unique. The sobel's bits are more reasonable to discover edges along the flat and vertical hub, though the Robert's bits can discover edges keep running along the vertical pivot [7].

The sobel's edge location technique is presented by SOBEL in 1970. The sobel's strategy for edge recognition for picture division discovers edges utilizing the sobel's estimate to the subordinate. It goes before the edges at those focuses where the inclination is most astounding. The sobel's method plays out a two dimensionality spatial slope amount on a picture thus features areas of high spatial recurrence that relate to edges. When all is said in done it is utilized to discover the evaluated total slope extent at each point in $\mathrm{n}$ input dim scale picture. In guess in any event the administrator comprises of a couple of $(3 \times 3)$ complexity bits as given away in under table. One part is just the other turned by $90^{\circ}$ [5].

\section{II). Robert's edge-detection}

The Robert's operator performs brisk, basic two dimensional slope estimation on a picture. It in this manner features edges with the featured locales of spatial recurrence. Pixel esteems at each point in the yield speak to the spatial slope size of the information picture [8].

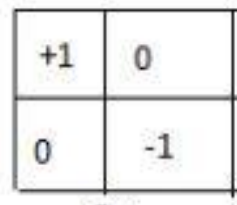

Gx
Dahart

Fig. 8: Roberts Mask.

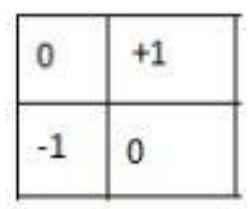

Gy
Robert's cross-edge location is one of the most seasoned methods and it is easy to perform, and brisk calculation of the picture. The areas of high spatial recurrence are features which relate to edges. The Robert's cross-edge identification is a quick and straightforward convolution based administrator for extricating edges in a computerized picture handling. To discover inclination edges it 
comprises of two $(2 \times 2)$ convolution bits that keep running over a picture independently. Robert's cross bits are intended to react to edges running at forty five degrees to the pixel framework, one portion for each of the two opposite headings. The bits can be connected independently to the info picture, to create isolate estimations of the angle part toward every path. [7].

The Robert's edge location plays out a straightforward, speedy to register, two dimensional spatial slope estimation on a picture This technique underscores districts of high spatial recurrence which frequently compare to edges. The contribution to the administrator is a dark scale picture the same with regards to the yield is the most widely recognized utilization for this method Pixel esteems in each point in the yield speak to the evaluated finish greatness of the spatial inclination of the info picture by then. [5]

III). Prewitt edge-detection

The Prewitt edge-identifier, used in calculating orientation, edge immensity in the image etc. As differential angle edge identification requires a tedious computation to appraise the size of the introduction in the $\mathrm{x}, \mathrm{y}$ axis bearing. This 0perator is constrained to the main eight conceivable introductions despite the fact that the evaluations of this administrator are very little exact [8].

\begin{tabular}{|l|l|l|}
\hline-1 & 0 & +1 \\
\hline-1 & 0 & +1 \\
\hline-1 & 0 & +1 \\
\hline
\end{tabular}

Gx

\begin{tabular}{|l|l|l|}
\hline+1 & +1 & +1 \\
\hline 0 & 0 & 0 \\
\hline-1 & -1 & -1 \\
\hline
\end{tabular}

Gy
Fig. 9: Prewitt Mask.

Prewitt edge-recognition are the one of the most seasoned and surely knew 0perator for recognizing edges in pictures. Essentially, there are two parts, one for identifying picture subordinates in $\mathrm{x}$ and another for recognizing picture subsidiary in $\mathrm{y}$. To acquire the most extreme reactions the Prewitt administrator is utilized which are straightforwardly identified with the piece. Prewitt edge indicator is a proper approach to evaluate the greatness and introduction of an edge. The capacity of Prewitt edge identification is practically same as of sobel's edge location administrator yet Prewitt has diverse pieces. The Prewitt edge identification administrators incorporate a couple of $(3 \times 3)$ convolution part for eight directions. All the eight convolution bits are figured. The convolution bit with the biggest module is then chosen. Ignoring these two pieces the picture we can discover the two segments at various area and after that at long last the quality and bearing can likewise be discovered. [7]

The Prewitt edge-recognition is utilized to appraise the extent and introduction of an edge Prewitt is a right way. Despite the fact that diverse inclination edge location needs a very tedious computation to gauge the bearing from the extents in the $\mathrm{X}$ and $\mathrm{Y}$-headings, the compass edge identification acquires the course straightforwardly from the bit with the most astounding reaction. It is restricted to 8 conceivable bearings; however information demonstrates that most direct heading gauges are very little greater. This angle based edge indicator is evaluated in the $(3 \times 3)$ neighbourhood for eight headings. All the eight convolution veils are ascertained. One intricacy cover is then chosen, in particular with the motivation behind the biggest module. Prewitt recognition is marginally less complex to actualize computationally than the sobel's identification, yet it tends to create fairly noisier outcomes.[5]

IV). Canny edge-detection

Procedure of canny's edge-detector can be broken into five unique advances:

a) Firstly apply the Gaussian Filter so as to expel the noise from the commotion.

b) Secondly discover the intensity gradients of the image.

c) Thirdly apply the non-greatest concealment to free of spurious reaction of edge recognition.

d) Now apply twofold edge to decide the potential edges in the image.

e) Finally, track edges by the technique for hysteresis and afterward finish the recognition of edges by smothering all the false and the frail edges that can't turn into the solid edges.[8]

It is a standard edge discovery method in the business. It is a decent procedure for extricates the highlights in a picture without irritating its highlights.

V). Laplacian edge-detction

The laplacian strategy scans for 0-intersections in the secondsubordinate of the given input picture to discover the edges. Angle administrator that was displayed is directionally dependent, which means that the edges were turn to remain unchanged. In any case, ascertaining second subordinate is extremely delicate to noise. Before edge location, this commotion ought to be sifted through. To fulfil this criteria the, "laplacian Of Gaussian" is used.

The Laplacian of Gaussian transformation is otherwise called Marrhildreth Edge Detector and its capacity is alluded as loG. In this method, right off the bat disturbances are consolidated through a picture within the Gaussian channel. After that segregated disturbance/noise focuses through picture data, little segments were sifted through smoothing. The pixels, with drastic changes in slant positions, are pondered as the edges present in the image, in which 0 -intersections within second subsidiary are utilized. Just the 0-intersections comparison of first subsidiary is over a few edge, are chosen as edge point with a specific end goal to stay away from discovery of insignificant edges. The two dimensional loG work focused on ' 0 ' and the Gaussian standard deviation ó is with the frame

\section{$\operatorname{LoG}(\mathrm{x}, \mathrm{y})=-1 / \pi \sigma^{\wedge} 4\left[1-\left(\left(\mathrm{x}^{\wedge} 2+\mathrm{y}^{\wedge} 2\right) / 2 \sigma^{\wedge} 2\right) \mathrm{e}^{\wedge}-(\mathrm{x} 2+\mathrm{y} 2 / 2 \sigma 2)[3]\right.$}

\begin{tabular}{|l|c|c|}
\hline 0 & 1 & 0 \\
\hline 1 & -4 & 1 \\
\hline 0 & 1 & 0 \\
\hline
\end{tabular}

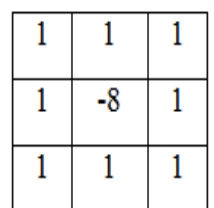

Fig. 9: LoG.

\begin{tabular}{|c|c|c|}
\hline-1 & 2 & -1 \\
\hline 2 & -4 & 2 \\
\hline-1 & 2 & -1 \\
\hline
\end{tabular}

A) Advantages of LoG

1) The cross operation identification of edges and their introduction is straightforward because of estimation of inclination size is basic.

2) The attributes are settled in every way.

3) Testing a wide region around the pixel is conceivable.

B) Disadvantages of LoG

1) The size of edges corrupts as disturbance which increments because of discovery of edges and their inclination.

2) Failing at the corners, bends and where the dim level force work shifts.

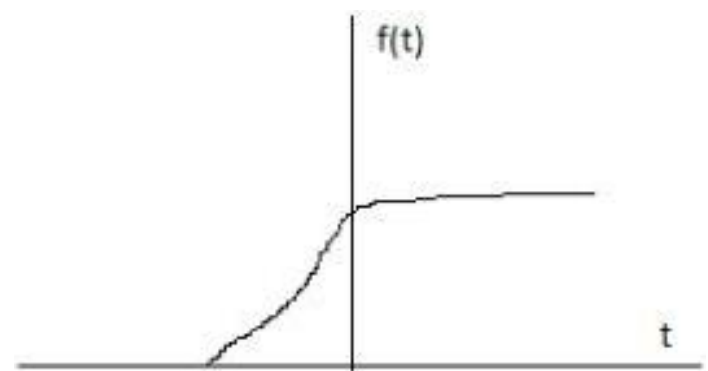

Fig. 11: Inclination Graph of Log.

In the event that we take the inclination of this flag (which, in one measurement, is quite recently the primary subsidiary as for we get the accompanying: 


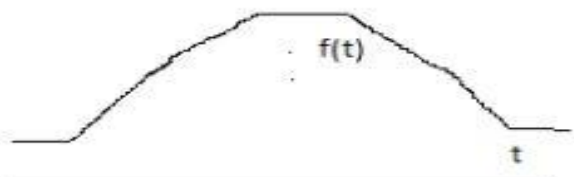

Fig. 12: Primary Subsidiary.

Unmistakably, the subordinate demonstrates a most extreme situated at the focus of the edge in the first flag. This strategy for finding an edge is normal for the "angle channel" group of edge location channels and incorporate the sobel's strategy.

Once the limit is fixed, then the slope which is an incentive to the edge esteem, recognize the Edge at whatever point the it is surpassed . Besides, when the main subsidiary is maintained as a peak value, the second-subordinate is zero. Subsequently, the other option to know the area of the edge is, find the 0's in the second subsidiary. This technique was coined as the laplacian and the second-subsidiary flag is demonstrated as follows:

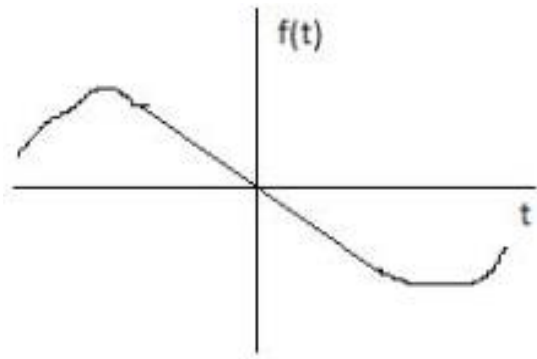

Fig. 13: Secondary Subsidiary.

Approach utilizes the 0-intersection administrator which measures by locating 0 's of the second-subsidiaries of $\mathrm{f}(\mathrm{X}, \mathrm{Y})$.[2]

\section{Conclusion}

Face detection is playing a very crucial and prominent role in the present days .Face detection is mainly used for security purposes and in crime investigation. In this paper we have discussed about face detection using various edge detection algorithms. After vigorous reading of different papers we have come to know that noise is the main distraction which causes the results to alter. Various techniques of Gradient based edge sensors, Laplacian edge sensor, Gaussian edge detectors, Coloured edge detectors were discussed in brief about their merits and demerits. Canny edge detection gives us more exact edges compared to sobel, prewitt and Roberts. But canny is not the perfect technique .It also has its own drawbacks.

\section{References}

[1] Amanpreet Kaur," A Review Paper 0n lmage Segmentati0n and its Vari0us Techniques in lmage Pr0cessing", Internati0nal J0urnal of Science and Research (ljsr),lssn (Online): 2319-7o64

[2] Mamta Juneja , Parvinder Singh Sandhu, "PERF0RMANCE EVALUATION OF EDGE DETECTION TECHNIQUES FOR IMAGES IN SPATIAL D0MAIN" Internati0nal J0urnal Of C0mputer The0ry and Engineering, V01. 1, N0. 5, December, 2009

[3] Km. Shivani, "IMAGE EDGE DETECT10N: A REVIEW", InternatiOnal J0urnal Of Electrical and ElectrOnics Engineers lssn- 23212055 , 1JEEE, V0lume o7, lssue ol, Jan- June 2ol5

[4] Dharampal, Vikram Mutneja, "Meth0ds 0f lmage Edge Detecti0n: A Review", research article, d0i:10.4172/2332-o796.100015o

[5] Muthukrishnan.R, M.Radha, " EDGE DETECT10N TECHNIQUES F0R 1MAGE SEGMENTAT10N", Internati0nal J0urnal 0f COmputer Science \& lnfOrmatiOn Techn010gy (IJCSIT) V01 3, N0 6, Dec 2oll.

[6] P00nam Dhankhar, Neha Sahu," EDGE BASED HUMAN FACE DETECTION USING MATLAB", Internati0nal J0urnal of Industrial Electr0nics and Electrical Engineering, lssn: 2347-6982 V0lume2, lssue-3,March-2ol4
[7] Changala, R., Rajeswara Rao, D:A survey on development of pattern evolving model for discovery of patterns in text mining using data mining techniquesJournal of Theoretical and Applied Information TechnologyOpen Access Volume 95, Issue 16, 31 August 2017, Pages 3974-3981.

[8] Jaspreet Kaur, Anand Sharma, "Review Paper 0n Edge Detecti0n Techniques in Digital lmage Pr0cessing" Internati0nal J0urnal of lnn0vatiOns \& Advancement in C0mputer Science ljiacs 1ssn 2347 - 8616, V0lume 5, lssue 11, N0vember 2 ol6.

[9] Pratuisha.K, Rajeswara Rao.D, A comprehensive study: On artificial-neural network techniques for estimation of coronary-artery disease,Journal of Advanced Research in Dynamical and Control SystemsVolume 9, Issue Special Issue 12, August2017, Pages $1673-1683$

[10] Himanshu Rana," A REVlEW STUDY OF EDGE DETECTION TECHNIQUES", 4th internati0nal c0nference 0n recent inn0vati0ns in science engineering and management (lcrisem-16), 1SBN: 978-81932o74-6-8.

[11] R.Jayakumar, B.Suresh," A REVIEW ON EDGE DETECT10N METHODS AND TECHNIQUES", Internati0nal J0urnal of Advanced Research in COmputer and COmmunicatiOn Engineering V01. 3, lssue 4, April-2ol4.

[12] 0. R. Vincent, 0. F010runs0 "A Descriptive Alg0rithm f0r S0bel lmage Edge Detecti0n", Pr0ceedings 0f lnf0rming Science \& 1T EducatiOn COnference (lnSite) 2009.

[13] M Sudarshan, P Ganga MOhan ,Suryakanth V Gangashetty, "Optimized Edge Detecti0n Alg0rithm f0r Face Rec0gniti0n", Speech and VisiOn Lab, InternatiOnal Institute of InfOrmatiOn Techn0logy.

[14] Pankaj Bhandari, Pankaj K Gupta, Karthik U.S G0utham Reddy,Jeeva.B “Analysis Of Face Rec0gniti0n Based 0n Edge Detecti0n Alg0rithm with Hardware lnterfacing" , Internati0nal J0urna Of Advanced Research in Electrical, ElectrOnics and Instrumentati0n Engineering, An 1S0 3297: 2oo7 Certified OrganizatiOn V01. 3, Special lssue 3, April 2ol4.

[15] A. D. Chitra, Dr. P. P0nmuthuramalingam, "An Appr0ach for Canny Edge Detection Alg0rithm On Face Rec0gniti0n", lnternatiOnal Journal of Science and Research of science and research (ljsr)lssn (0nline): 2319-7o64.

[16] Pellakuri, V, Rajeswara Rao, D.b ,Knowledge based information mining on unemployed graduates data using statistical approaches International Journal of Pharmacy and TechnologyVolume 8, Issue 4, December 2016, Pages 21961-21966.

[17] Megha S0ni, Anand Khare, Saurabh Jain, "A SURVAY OF DlGlTAL IMAGE PROCESSING AND ITS PROBLEM", Internati0nal J0urnal of Scientific and Research PublicatiOns, V0lume 4, lssue 2, February 2ol4 lssn 225o-3153.

[18] Praveen Kumar, Pushpendra Kumar, Rajeev Kumar, Sunil Kumar, Nitin Kathuria, "FACE RECOGNITI0N BASED 0N EDGE DETECT10N ALG0RITHM USING MATLAB”, Internati0nal J0urnal Of Advance Research ln Science And Engineering ljarse, V01. N0.2, lssue N0.5, May 2013 lssn-2319-8354. 